Further

$v(r, \theta)=\frac{1}{\pi} \int_{-\pi}^{\pi} K(r, \theta-x) V(1, x) d x=\frac{1}{\pi} \int_{\alpha}^{\beta} K(r, \theta-x) V(1, x) d x$

is clearly a function, harmonic in $r<1$ and it is easy to deduce from the properties of $K(r, \theta)$ and $V(1, \theta)$ that $v(r, \theta)$ satisfies all the conditions of the theorem.

COROLlary. If $f(z)$ is analytic in $|z|<1$, then, given the arc $(|z|=1$, $\alpha<\arg z<\beta)$, there is a function $g(z)$ analytic in $|z|<1$ and on the arc of $|z|=1$ complementary to $(\alpha, \beta)$, such that $f(z)-g(z)$ can be extended analytically across $(\alpha, \beta)$.

University OF CALIFornia

\title{
ON THE COMPLEX ZEROS OF THE BESSEL FUNCTIONS
}

E. HILLE AND G. SZEGÖ

1. Introduction. Various proofs have been given for the following classical theorem of A. Hurwitz:

THEOREM 1. The entire function

$$
z^{\beta / 2} J_{-\beta}\left(2 z^{1 / 2}\right)=\sum_{m=0}^{\infty} \frac{(-z)^{m}}{m !} \frac{1}{\Gamma(m+1-\beta)}
$$

has precisely $[\beta]$ nonpositive zeros. Here $J_{-\beta}$ is the Bessel function of order $-\beta$ and $\beta \geqq 0$.

In case $\beta$ is an integer these nonpositive zeros are all at the origin; in case $\beta$ is not an integer and $[\beta]$ is odd there is precisely one negative zero and we have $\frac{1}{2}([\beta]-1)$ pairs of conjugate complex zeros; in case $\beta$ is not an integer and $[\beta]$ is even there are $\frac{1}{2}[\beta]$ pairs of conjugate complex zeros.

Most of the proofs for this theorem (see the papers $[2,4,6,7,9]$ of the Bibliography at the end of the text) make use of polynomial approximations of the Bessel function. The present proof follows the same line by obtaining the Bessel function as the limit of Laguerre polynomials. The study of the complex zeros of these polynomials is 1942.

Presented to the Society, September 9, 1942; received by the editors September 14, 
particularly simple and for this reason the present arrangement may have certain advan tages. ${ }^{1}$ Our proof yields also certain bounds for the nonpositive zeros of the function (1.1) in terms of $\beta$.

In $\S 2$ we give a compilation of the definitions and results on Laguerre polynomials which are essential for our proof. We follow the notation of G. Szegö, Orthogonal polynomials (Amer. Math. Soc. Colloquium Publications, vol. 23, New York, 1939; quoted as Sz.).

$\$ 3$ contains certain bounds for the nonpositive zeros of Laguerre polynomials from which Theorem 1 follows immediately.

$\$ 4$ gives bounds for the nonpositive zeros of the function (1.1).

In $\S 3$ we use as an important instrument an elementary formula involving a solution of a second order linear differential equation which was employed for the study of the zeros of various functions by A. Hurwitz and E. Hille [5, 3].

2. Laguerre polynomials. We define the Laguerre polynomials by [Sz. (5.1.6)]

$$
L_{n}^{(\alpha)}(x)=\sum_{m=0}^{n} C_{n+\alpha, n-m} \frac{(-x)^{m}}{m !} .
$$

This is equivalent to the formula [Sz. (5.1.5)]

$$
e^{-x} x^{\alpha} L_{n}^{(\alpha)}(x)=(n !)^{-1}(d / d x)^{n}\left(e^{-x} x^{n+\alpha}\right) .
$$

It is easy to verify the differential equation [Sz. (5.1.2)]

$$
\left\{\begin{array}{c}
w^{\prime \prime}+G(x) w=0, \quad w=w(x)=e^{-x / 2} x^{(\alpha+1) / 2} L_{n}^{(\alpha)}(x), \\
G(x)=A x^{-1}-B x^{-2}-1 / 4, \quad A=n+(\alpha+1) / 2, \\
B=\left(\alpha^{2}-1\right) / 4 .
\end{array}\right.
$$

Let $x=u+i v \neq 0$; we note that

$$
\Im\{G(x)\}=v\left\{-A\left(u^{2}+v^{2}\right)^{-1}+2 B u\left(u^{2}+v^{2}\right)^{-2}\right\} .
$$

Finally we conclude from (2.1) by comparing the corresponding powers of $x$ [Sz. (5.1.14)]

$$
(d / d x) L_{n}^{(\alpha)}(x)=-L_{n-1}^{(\alpha+1)}(x) .
$$

1 The proof given by Pólya [7] deduces Theorem 1 from certain theorems of a general character. It may be of interest to observe that his theorem on the so-called Jensen polynomials [7, p. 165] furnishes the result of Hurwitz; indeed, the Jensen polynomials associated with the function (1.1) are (except for a factor independent of $x$ ) the Laguerre polynomials $L_{n}^{(-\beta)}(x)$. 
LEMMA 1. Let $n \geqq 1, \alpha$ an arbitrary real number, $\alpha \neq-1,-2, \cdots,-n$. Then the zeros of $L_{n}^{(\alpha)}(x)$ are not equal to 0 and distinct. Furthermore the number of the positive zeros is $n$ if $\alpha>-1$; it is $n+[\alpha]+1$ if $-n<\alpha<-1$; it is 0 if $\alpha<-n$. The number of the negative zeros is 0 or 1. [Sz., Theorem 6.73.]

Let $\alpha=-\beta$ be negative, $\beta$ not an integer. If $n>\beta$ the polynomial $L_{n}^{(-\beta)}(x)$ has $[\beta]$ nonpositive zeros which are all complex, except a single negative one in case $[\beta]$ is odd.

This theorem is due to E. Stridsberg [8] and W. Hahn [1]. It follows from older results on Jacobi polynomials by a limiting process [Sz. loc. cit.]. A continuity proof based on the variation of $\beta$ is also possible.

For the sake of completeness we give here the following very simple proof of Lemma 1.

From (2.1) and (2.3) we conclude that all the zeros are not equal to 0 and distinct. In case $\alpha>-1$ Rolle's theorem applied to formula (2.2) furnishes exactly $n$ positive zeros. Further let $-k-1<\alpha<-k$ $(1 \leqq k \leqq n)$. The same theorem furnishes then at least $n-k$ positive zeros, and by Descartes' rule of signs we obtain exactly this number of positive zeros. Inspection of (2.1) shows that for $k \geqq n$ no positive zeros exist. Now let $x$ be negative and $\alpha<-1$. Then ${ }^{2} G(x)<0$ so that $w$ and $w^{\prime \prime}$ are of the same sign. This excludes the possibility of two negative zeros. Hence the number of the negative zeros is 0 or 1 .

LEMMA 2. Let $\alpha$ be an arbitrary real number. Then

$$
\lim _{n \rightarrow \infty} n^{-\alpha} L_{n}^{(\alpha)}(z / n)=z^{-\alpha / 2} J_{\alpha}\left(2 z^{1 / 2}\right)
$$

uniformly if $z$ is bounded. [Sz. Theorem 8.1.3.]

This follows immediately from (2.1).

In view of the limit formula (2.6)Hurwitz's theorem results, provided we can show that the nonpositive zeros of $L_{n}^{(\alpha)}(x)$ are in absolute value not greater than $C n^{-1}$, where $C=C(\alpha)$ is independent of $n$. (Indeed, the zeros of the functions on the left-hand side of (2.6) tend to those of the function on the right-hand side, as $n \rightarrow \infty$.) The only possibility which has to be excluded is the confluence of two conjugate complex zeros of $L_{n}^{(\alpha)}(x)$ into a real zero of $z^{-\alpha / 2} J_{\alpha}\left(2 z^{1 / 2}\right)$, which would be necessarily multiple and not equal to 0 . But this is out of the question since this function as a solution of a second order linear differ-

${ }^{2} G(x) \leqq(\alpha+1) / 2 x-\left(\alpha^{2}-1\right) / 4 x^{2}-1 / 4 \leqq(1 / 2) /(\alpha-1)<0$. 
ential equation with the only finite singularity $z=0$ can not have a multiple zero different from 0 .

3. Bounds for the nonpositive zeros of $L_{n}^{(\alpha)}(x)$. In what follows we use the notation of $\$ 2$.

LEMMA 3. Let $\alpha=-\beta$ be negative, $\beta$ not an integer and $[\beta]$ odd. Then for the only negative zero $x_{0}$ of $L_{n}^{(-\beta)}(x)$ we have

$$
x_{0}>-\beta^{2} / 4 n
$$

provided $n$ is sufficiently large.

According to $(2.5) L_{n}^{(-\beta)}(x)$ is monotonic for negative $x$; furthermore $L_{n}^{(-\beta)}(0)<0$. Thus $x_{0}>-c n^{-1}, c>0$, provided $L_{n}^{(-\beta)}\left(-c n^{-1}\right)>0$. Since, $n>[\beta]$,

$$
\begin{aligned}
L_{n}^{(-\beta)}\left(-c n^{-1}\right) & =\sum_{m=0}^{[\beta]} C_{n-\beta, n-m} \frac{\left(c n^{-1}\right)^{m}}{m !}+\sum_{m=[\beta]+1}^{n} C_{n-\beta, n-m} \frac{\left(c n^{-1}\right)^{m}}{m !} \\
& >\sum_{m=0}^{[\beta]} C_{n-\beta, n-m} \frac{\left(c n^{-1}\right)^{m}}{m !}
\end{aligned}
$$

and

(3.3) $\lim _{n \rightarrow \infty} n^{\beta} \sum_{m=0}^{[\beta]} C_{n-\beta, n-m} \frac{\left(c n^{-1}\right)^{m}}{m !}=\sum_{m=0}^{[\beta]} \frac{c^{m}}{m !} \frac{1}{\Gamma(m+1-\beta)}$,

we have $L_{n}^{(-\beta)}\left(-c n^{-1}\right)>0$ for sufficiently large $n$ provided the expression on the right-hand side of (3.3) is positive. This is the case if $c$ is chosen sufficiently large.

It is easy to specify the constant $c$. The above expression is certainly positive provided

$$
c>m(\beta-m), \quad m=1,2,3, \cdots,[\beta] .
$$

We can take, for instance,

$$
c=\beta^{2} / 4 \text {. }
$$

The formula of A. Hurwitz and E. Hille mentioned in the introduction applied to (2.3) will read as follows:

LEMмA 4. Let $a$ and $b$ be arbitrary complex numbers, $a \neq b, a \neq 0$, $b \neq 0$. Then

$$
\left[\bar{w} w^{\prime}\right]_{a}^{b}-\int\left|w^{\prime}\right|^{2} \overline{d x}+\int G(x)|w|^{2} d x=0 ;
$$

the integration is extended over an arbitrary rectifiable curve in the com- 
plex $x$-plane joining $a$ and $b$ and avoiding the origin.

This furnishes immediately a bound for the imaginary parts of the zeros of $L_{n}^{(\alpha)}(x)$. More exactly we prove:

Lemma 5. Let $\alpha=-\beta$ be negative, $\beta$ not an integer, $n>\beta>1$. For any complex zero $p+i q$ of $L_{n}^{(-\beta)}(x)$ we have

$$
|q| \leqq B / A \text {. }
$$

Let $q \neq 0$; we apply (3.6), integrating from $p+i q$ to $\infty$ along a straight line parallel to the real axis with $\Re x \rightarrow+\infty$. Then, noting that $\overline{w(x)} w^{\prime}(x) \rightarrow 0$ when $x=u+i q, u \rightarrow+\infty$,

$$
-\int_{p+i q}^{\infty}\left|w^{\prime}\right|^{2} d x+\int_{p+i q}^{\infty} G(x)|w|^{2} d x=0
$$

follows, so that $\Im\{G(x)\}$ must vanish somewhere along this path. Thus we must have $-A\left(u^{2}+v^{2}\right)+2 B u=0$ for a suitable $u, u \geqq p, v=q$. This is the equation of a circle with center at $(B / A, 0)$ and radius $B / A$. From this (3.7) follows.

LeмmA 6. Let $\alpha=-\beta$ be negative, $\beta$ not an integer, $n>\beta>1$. Then for the complex zeros $p+i q$ of $L_{n}^{(-\beta)}(x)$ we have

$$
|p| \leqq \begin{cases}2 B / A & \text { if } p>0, \\ \left|x_{0}\right| & \text { if } p<0,\end{cases}
$$

where $x_{0}$ is the only negative zero of $L_{n}^{(-\beta)}(x)$ or $L_{n+1}^{(-\beta-1)}(x)$ depending upon whether $[\beta]$ is odd or even.

The previous argument furnishes the first part of the assertion. As to the second part, we assume that $[\beta]$ is odd; the case in which $[\beta]$ is even can be settled by means of Gauss' theorem on account of the relation

$$
(d / d x) L_{n+1}^{(-\beta-1)}(x)=-L_{n}^{(-\beta)}(x) .
$$

Let us assume that $p<x_{0}, q>0$. We apply (3.6) with $a=p, b=p+i q$ choosing for the path of integration a vertical segment. Then

$$
-\overline{w(p)} w^{\prime}(p)-\int_{p}^{p+i q}\left|w^{\prime}\right|^{2} \overline{d x}+\int_{p}^{p+i q} G(x)|w|^{2} d x=0
$$

follows. We write

$$
\mathfrak{w}(x)=e^{-x / 2}(-x)^{(\alpha+1) / 2} L_{n}^{(\alpha)}(x)
$$

and take the real parts of the terms in (3.9): 
(3.11) $-\{\mathfrak{w}(p)\}^{2} \frac{\mathfrak{w}^{\prime}(p)}{\mathfrak{w}(p)}-\int_{p}^{p+i q}\{\Im G(x)\}|\mathfrak{w}(x)|^{2}|d x|=0$.

Now $\mathfrak{w}(x)$ satisfies the differential equation (2.3) and $G(x)<0$ for $x<0$. Hence $\mathfrak{w}^{\prime \prime}(x)$ and $\mathfrak{w}(x)$ are of the same sign, that is $\mathfrak{w}^{\prime \prime}(x)>0$ for $x<x_{0}$. Thus $\mathfrak{w}^{\prime}(x)$ is increasing for $x<x_{0}$ and since $\mathfrak{w}^{\prime}\left(x_{0}\right)$ $=e^{-x_{0} / 2}\left(-x_{0}\right)^{(\alpha+1) / 2} L_{n}^{(\alpha) \prime}\left(x_{0}\right)<0$ we have $\mathfrak{w}^{\prime}(x)<0$ for $x<x_{0}$. Consequently the first term of (3.11) is positive. But according to (2.4) $\Im\{G(x)\}<0$ so that (3.11) involves a contradiction.

4. Conclusion. From the Lemmas 3,5 and 6 we deduce Theorem 1 in a familiar way (cf. the end of $\$ 2$ ). By using (3.1), (3.7) and (3.8) we find more precisely:

TheOREM 2. Let $\alpha=-\beta$ be negative, $\beta$ not an integer, $\beta>1$. The nonpositive zeros of the function $z^{\beta / 2} J_{-\beta}\left(2 z^{1 / 2}\right)$ are situated in the rectangle

$$
\left.\begin{array}{l}
-\beta^{2} / 4 \\
-(\beta+1)^{2} / 4
\end{array}\right\} \leqq \Re z \leqq\left(\beta^{2}-1\right) / 2 ; \quad|\Im z| \leqq\left(\beta^{2}-1\right) / 4 .
$$

In the first inequality the upper or lower expression holds depending on whether $[\beta]$ is odd or even.

\section{BIBLIOGRAPHY}

1. W. Hahn, Die Nullstellen der Laguerreschen und Hermiteschen Polynome, Schriften des Mathematischen Seminars und des Instituts für angewandte Mathematik der Universität Berlin, vol. 1 (1933) pp. 213-244.

2. E. Hilb, Die komplexen Nullstellen der Besselschen Funktionen, Math. Zeit. vol. 15 (1922) pp. 274-279.

3. E. Hille, Oscillation theorems in the complex domain, Trans. Amer. Math. Soc. vol. 23 (1923) pp. 350-385.

4. A. Hurwitz, Über die Nullstellen der Bessel'schen Funktion, Math. Ann. vol. 33 (1889) pp. 246-266. See Mathematische Werke, vol. 1, 1932, pp. 266-286.

5. - Über die Nullstellen der hypergeometrischen Funktion, Math. Ann. vol. 64 (1907) pp. 517-560. See Mathematische Werke, vol. 1, 1932, pp. 660-705, in particular pp. 700-704.

6. N. Obreschkoff, Über die Nullstellen der Besselschen Funktionen, Jber. Deutschen Math. Verein. vol. 38 (1929) pp. 156-161.

7. G. Pólya, Über einen Satz von Laguerre, Jber. Deutschen Math. Verein. vol. 38 (1929) pp. 161-168.

8. E. Stridsberg, Nägra aritmetiska undersökningar rörande fakulteter och vissa allmännare Koefficientsviter, Not 2, Arkiv för Matematik, Astronomi och Fysik, vol. 13 no. 25 (1918) 70 pp., in particular pp. 34-35.

9. G. N. Watson, The zeros of Lommel's polynomials, Proc. London Math. Soc. (2) vol. 19 (1921) pp. 266-272.

YALE UNIVERSITY AND

STANFORD UNIVERSITY 